\title{
Association of the GSTM1 null polymorphism with breast cancer in a Mexican population
}

O. Soto-Quintana ${ }^{1,2}$, G.M. Zúñiga-González ${ }^{3}$, R. Ramírez-Patiño ${ }^{1,4}$, A. Ramos-Silva1,2, L.E. Figuera ${ }^{5}$, D.I. Carrillo-Moreno ${ }^{1,4}$, I.A. Gutiérrez-Hurtado ${ }^{1,4}$, A.M. Puebla-Pérez ${ }^{6}$, B. Sánchez-Llamas ${ }^{7}$ and M.P. Gallegos-Arreola ${ }^{1}$

${ }^{1}$ Molecular Genetics Laboratory, Molecular Medicine Division, Western Biomedical Research Center, Western National Medical Center, Mexican Institute of Social Security, Guadalajara, Jalisco, Mexico 2Doctorate Program in Pharmacology, Health Sciences University Center, University of Guadalajara, Guadalajara, Jalisco, Mexico ${ }^{3}$ Mutagenesis Laboratory, Molecular Medicine Division, Western Biomedical Research Center, Western National Medical Center, Mexican Institute of Social Security, Guadalajara, Jalisco, Mexico ${ }^{4}$ Doctorate Program in Human Genetics, Health Sciences University Center, University of Guadalajara, Guadalajara, Jalisco, México, ${ }^{5}$ Genetics Division, Western Biomedical Research Center, Western National Medical Center, Guadalajara, Jalisco, México ${ }^{6}$ Immunopharmacology Laboratory, Exact Science and Engineer University Center, University of Guadalajara, Guadalajara, Jalisco, México

${ }^{7}$ Highly Specialized Medical Unit of Gynecology-Obstetrics Hospital, Western National Medical Center, Mexican Institute of Social Security, Guadalajara, Jalisco, México

Corresponding author: M.P. Gallegos-Arreola E-mail: marthapatriciagallegos08@gmail.com

Genet. Mol. Res. 14 (4): 13066-13075 (2015)

Received May 15, 2015

Accepted July 31, 2015

Published October 26, 2015

DOI http://dx.doi.org/10.4238/2015.October.26.2

ABSTRACT. The glutathione $S$ transferase (GST) family plays an important role in the processing of carcinogens. Data on the null GSTM1 genotype has revealed associations with cancer, and has been suggested to affect carcinogen metabolism and to contribute to tumor promotion in 
the mammary gland. We examined the role of the null GSTM1 genotype by comparing the genotypes of 276 healthy Mexican women with those of 558 Mexican women with breast cancer (BC). The genotype frequencies observed in the controls and patients with $\mathrm{BC}$ were 38 and $45 \%$ for the null GSTM1 genotype, respectively. The obtained odds ratio (OR) was 1.36 , with a $95 \%$ confidence interval $(95 \% \mathrm{Cl})$ of $1.02-1.8, P=0.04$. The protective association was also evident upon analysis of the distributions of the null GSTM1 genotype in patients with positive chemotherapy response who had high plasma levels of glucose $(\mathrm{OR} 0.56,95 \% \mathrm{Cl}=0.33-0.94, \mathrm{P}=$ 0.03). This study suggested that the null GSTM1 genotype is associated with BC susceptibility in the Mexican population analyzed.

Key words: GSTM1 polymorphism; Breast cancer; Chemotherapy response; Mexican population

\section{INTRODUCTION}

Breast cancer $(\mathrm{BC})$ is one of the most common diseases in developing countries and around the world, although the incidence rates of this disease vary in different ethnic groups (Miller et al., 2012; Siegel et al., 2012). In many countries, particularly in Mexico, the incidence of BC has increased in the last seven years, such that $B C$ is now one of the main causes of death in working-age women (Chávarri-Guerra et al., 2012). The state of Jalisco exhibits one of the highest mortalities associated with BC, and only $10 \%$ of all cases of $\mathrm{BC}$ are detected early at stage I (Gómez Flores-Ramos et al., 2013; Ramírez-Patiño et al., 2013; Gallegos-Arreola et al, 2014). BC is considered to be a multifactorial disease and might result from a combination of abnormal gene interactions and environmental factors (Gómez Flores-Ramos et al., 2013; Gallegos-Arreola et al., 2014). Therefore, elucidating genetic variants among different ethnic groups could contribute to an explanation for the differences in the progression of cancer as well as in chemotherapeutic response between these groups.

Determination of the relationships between the detoxification of a wide variety of known and suspected carcinogens and the susceptibility to cancers including BC is a growing field of research (de Aguilar et al., 2012). The family of glutathione S-transferase (GST) cytosolic enzymes is involved in biotransformation phase II and acts on carcinogens, environmental pollutants, chemotherapeutic drugs, and other xenobiotics. This family is composed of six classes of dimeric isoenzymes (GST alpha, mu, pi, theta, zeta, and omega) that have been implicated as key detoxification enzymes. GST mu (GSTM1) is expressed in the liver, kidney, and lung and is involved in the detoxification of polycyclic aromatic hydrocarbons and other carcinogens. Individuals with the GSTM1 null genotype are more susceptible to DNA damage than are wild type individuals (de Aguilar et al., 2012; Duggan et al., 2013). The GSTM1 gene is located on chromosome 1p13.3, contains 10 exons, is polymorphic in humans, and possesses three known alleles: GSTM1*A, GSTM1 ${ }^{*} B$, and $G S T M 1{ }^{*} O$ (or null). The GSTM1 ${ }^{*} A$ (containing a lysine at position number 172 of the protein), and ${ }^{*} B$ (containing asparagine at this position) alleles differ only at a single amino acid (K172N) and appear to be functionally identical (McLellan et al., 1997).

The most common variant of GSTM1 is the null genotype, which has been shown to be present in 20 to $50 \%$ of the population and lacks detectable expression of the gene product 
(Duggan et al., 2013). This leads to a lack of expression of the GST mu enzymes that results in decreased antioxidant activity in the cell (Duggan et al., 2013), high accumulation of reactive oxygen species (ROS), and consequently higher susceptibility to carcinogenic events due to DNA damage (McLellan et al., 1997; Wang et al., 2010). It has been suggested that the null GSTM1 is associated with an increased risk of developing some types of cancers including BC (McLellan et al., 1997; de Aguiar et al., 2012; Duggan et al., 2013; Possuelo et al., 2013; Wan et al., 2014). Wan et al. (2014), in a meta-analysis study, demonstrated an association of the null GSTM1 genotype with BC in a Chinese population. Possuelo et al. (2013) found an association between the GSTM1 null genotype with BC in a Brazilian population. Sohail et al. (2013) similarly demonstrated an increased susceptibility to BC in null GSTM1 members of a Pakistani population.

Several reports in the general Mexican population have described a high frequency of the null GSTM1 allele of approximately of $37-44 \%$, depending on the tested region (Pérez-Morales et al. 2011; Martinez-Ramirez et al., 2013; Sandoval-Carrillo et al., 2014). However, there are no descriptions to date of an association of the null GSTM1 polymorphism with BC in Mexican women.

\section{MATERIAL AND METHODS}

\section{Sample collection and patient information}

DNA was extracted from peripheral blood leucocytes from blood samples collected from 276 healthy women recruited as volunteer blood donors using standard protocols (Miller et al., 1988). These volunteers were not matched by age with the patient group. Blood samples were also collected from 558 patients with a clinical and histological diagnosis of BC between June 2010 and April 2014. All of the individuals included in this study were residents of the metropolitan area of Guadalajara. All samples were obtained after a written informed consent form was signed, which was previously approved by ethical committee 1301, Western National Medical Center, Mexican Institute of Social Security, Guadalajara, Jalisco, Mexico. This study was conducted respecting national and international ethical standards. Efforts were made to ensure that siblings of individuals who had already been sampled were excluded. Clinical and demographic data were obtained using written questionnaires. All patients were also interviewed to determine their occupational exposure and as well as use of pharmacological therapies. The BC patient database and the DNA samples have been examined for other polymorphisms (Gómez Flores-Ramos et al., 2013; Ramírez-Patiño et al., 2013; Gallegos et al, 2014).

\section{Genotyping}

A multiplex PCR method was used to detect the presence or absence of the GSTM1 gene in the genomic DNA samples of the study groups. Determination of the null GSTM1 polymorphism was performed using the following primers (exons 4-5): 5'-CTG CCC TAC TTG ATT GAT GGG3' and 5'-CTG GAT TGT AGC AGA TCA TGC-3', and amplification of the internal control was performed using the following primers: CFRT - EX4 5'-AGT CAC CAA AGC AGT ACA GC-3' and 5'-GCT ATT CTC ATC TGC ATT CC-3' (Baranova et al., 1997). The polymerase chain reaction (PCR) amplifications were performed in a $15 \mu \mathrm{L}$ total volume containing $0.2 \mathrm{mM}$ dNTPs (Invitrogen, Carlsbad, CA, USA), $7.5 \mathrm{pmol}$ primers, $2.5 \mathrm{mM} \mathrm{MgCl}_{2}, 2.5 \cup$ Taq polymerase (Invitrogen), and 50 ng genomic DNA. The PCR conditions were as follows: $94^{\circ} \mathrm{C}(4 \mathrm{~min})$, followed by 35 cycles of 
$94^{\circ} \mathrm{C}(50 \mathrm{sec}), 62^{\circ} \mathrm{C}(1 \mathrm{~min})$ and $72^{\circ} \mathrm{C}(1 \mathrm{~min})$, with a final extension at $72^{\circ} \mathrm{C}(7 \mathrm{~min})$. Using this procedure, two fragments of $271 \mathrm{bp}$ (indicating the presence of GSTM1, "no null") and $189 \mathrm{bp}$ (internal control) were obtained. Genotypic discrimination was performed using $6 \%$ polyacrylamide gel (29:1) electrophoresis, followed by silver staining (Figure 1) (Sanguinetti et al., 1994).

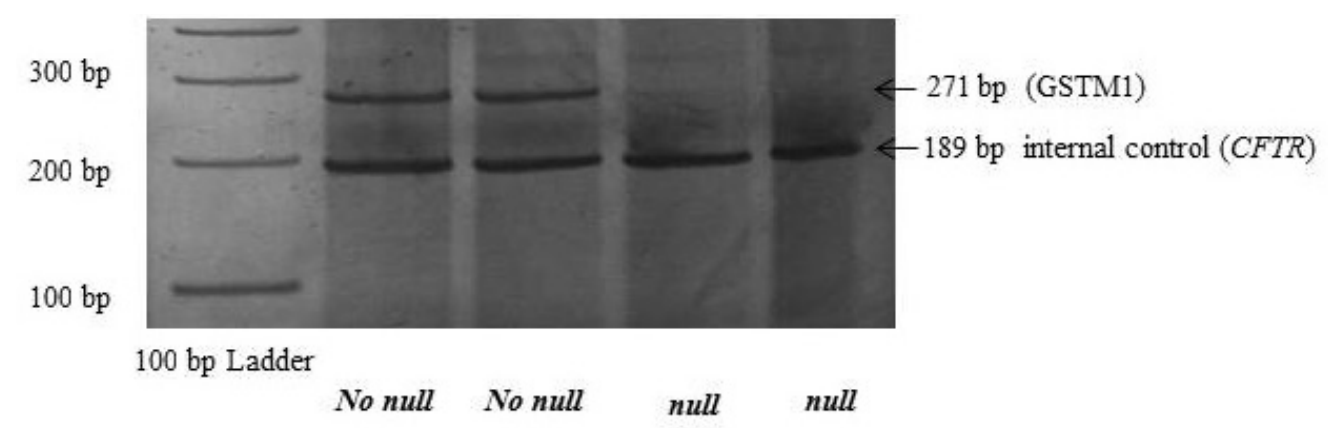

Figure 1. Polyacrylamide gel electrophoresis (6\% (29:1)) detection of internal control (189 bp; CFTR exon 4) and GSTM1 null (band absence) and "no null" (271 bp) genotypes.

\section{Statistical analysis}

Genotypic frequencies were obtained by direct counting and comparison by Chi square test among the study groups. Odds ratios and $95 \%$ confidence intervals $(\mathrm{Cls})$ were also calculated. A two-sided $P<0.05$ was considered to be statistically significant. All statistical analyses were performed using PASW Statistic Base 18 software, 2009 (Chicago, IL, USA).

\section{RESULTS}

The comparative demographic data from the patients with $\mathrm{BC}$ and the control individuals are shown in Table 1. In the patient group, the average age was 54.17 years, ranging from 25 to 88 years. Menarche presented at a mean age of 12.63 years in the patients and 12.09 years in the controls. Positive familial history of diabetes-arterial hypertension (DM2-AH) in first and second degree relatives of patients was observed to be risk factor (adjusted OR 1.8, $95 \% \mathrm{Cl}=1.3-2.6, \mathrm{P}=0.001$ ).

The clinical characteristics of the patients with $\mathrm{BC}$ included in the analysis were: body mass index (BMI: 18.5-24.9, $\geq 25-29.9, \geq 30-34.9$, and $\geq 35$ ) (WHO Expert Consultation, 2004); tumor markers (luminal A, B, Her1/neu, and triple negative); histology (ductal, lobular); chemotherapy type (FEC-capecitabine: 5-fluorouracil-epirubicin-cyclophosphamide; others (paclitaxel, docetaxel, Herceptin); and no chemotherapy); and laboratory test: (hemoglobin, hematocrit (HTO), platelets, leukocytes, glutamate-oxaloacetate transaminase (SGOT), glutamic pyruvic transaminase (SGPT), alkaline phosphatase (ALP), gamma glutamyl transferase (GGT), and glucose). Table 2 presents the results of the multivariate logistic regression analysis from data included in the Table 1 and the clinical characteristics of $B C$ listed above, where the $B C$ group was classified using menopause status as the dependent variable. A lobular carcinoma ( $\mathrm{OR} 2.3,95 \% \mathrm{Cl}=1.1-4.8, \mathrm{P}=0.022)$, presence of menarche $(14-18$ years) $(\mathrm{OR} 1.6,95 \% \mathrm{Cl}=1.03-2.5, \mathrm{P}=0.034)$, luminal $\mathrm{B}$ type (OR $1.9,95 \% \mathrm{Cl}=1.1-3.2, \mathrm{P}=0.017)$, and $\mathrm{DM} 2-\mathrm{AH}$ as personal medical history $(\mathrm{OR} 2.6,95 \% \mathrm{Cl}=1.6-$ $4.3, P=0.000$ ) were found to be risk factors associated with menopause status. 


\begin{tabular}{|c|c|c|c|c|}
\hline & Patients with $\mathrm{BC}(\mathrm{N}=558)$ & Controls $(N=276)$ & OR $(95 \% \mathrm{Cl})^{*}$ & $P$ value \\
\hline \multicolumn{5}{|l|}{ Age (years) } \\
\hline Mean (SD) & $54.17(11.63)$ & $35.31(8.86)$ & & $<0.0001$ \\
\hline \multicolumn{5}{|l|}{ Menarche (years) } \\
\hline Mean (SD) & $12.63(1.67)$ & $12.09(0.82)$ & & $<0.0001$ \\
\hline \multicolumn{5}{|l|}{ Menarche (range) } \\
\hline $7-10$ & (42) 8 & (10) 4 & & \\
\hline $11-13$ & (350) 62 & (248) 90 & & \\
\hline $14-18$ & (166) 30 & (18) 6 & & \\
\hline \multicolumn{5}{|l|}{ Tobacco consumption } \\
\hline Yes & (149) 27 & (61) 22 & & NS \\
\hline No & (409) 73 & (215) 78 & & \\
\hline \multicolumn{5}{|l|}{ Alcohol consumption } \\
\hline Yes & (105) 19 & (52) 18 & & NS \\
\hline No & (453) 81 & (264) 82 & & \\
\hline \multicolumn{5}{|l|}{ Family history (FH) } \\
\hline No & (167) 28 & (183) 66 & & \\
\hline $\mathrm{BC}$ & (73) 13 & (8) 3 & & \\
\hline DM2-AH & (132) 24 & (83) 30 & $1.8(1.3-2.6)$ & 0.001 \\
\hline DM2-AH-Cancer** & (196) 35 & (2) 1 & & \\
\hline
\end{tabular}

$\mathrm{BC}=$ breast cancer; $\mathrm{SD}$ = standard deviation; $\mathrm{NS}$ = no significant difference; $\mathrm{DM} 2-\mathrm{AH}$ = diabetes mellitus type 2-arterial hypertension; $\mathrm{OR}=$ odds ratio; $\mathrm{Cl}$ = confidence interval; * $\mathrm{OR}$ from the adjusted regression analysis; ** positive familial history of cancer and leukemia in first and second degree relatives of patients.

The genotype frequencies of the null GSTM1 allele were different in the control and patient groups (Table 3). The polymorphic genotype null GSTM1 was observed in $45 \%(252 / 558)$ of the patients with BC and $38 \%(104 / 276)$ of the controls (OR 1.3, 95\%Cl $=1.02-1.8, \mathrm{P}=0.04)$. All of the samples were analyzed, and all of the participant genotypes (for 276 controls and 558 patients with BC) were obtained.

\begin{tabular}{|c|c|c|c|c|c|c|c|c|}
\hline & \multirow[t]{2}{*}{ B } & \multirow[t]{2}{*}{ SD } & \multirow[t]{2}{*}{ Wald } & \multirow[t]{2}{*}{ d.f. } & \multirow[t]{2}{*}{$\mathrm{P}$} & \multirow[t]{2}{*}{ OR } & \multicolumn{2}{|c|}{$95 \% \mathrm{Cl}$} \\
\hline & & & & & & & Lower & Upper \\
\hline Lobular carcinoma & 0.84 & 0.36 & 5.2 & 1 & 0.022 & 2.3 & 1.1 & 4.8 \\
\hline Menarche (14-18 years) & 0.49 & 0.23 & 4.5 & 1 & 0.034 & 1.6 & 1.0 & 2.5 \\
\hline Luminal B & 0.64 & 0.27 & 5.6 & 1 & 0.017 & 1.9 & 1.1 & 3.2 \\
\hline DM2-AH* & 0.97 & 0.25 & 14.4 & 1 & 0.000 & 2.6 & 1.6 & 4.3 \\
\hline
\end{tabular}

Variables included in the analysis: dependent: BC classified by menopause or pre-menopause status; independent: *personal medical history, menarche ranging from 7-10 years, 11-13 years, or 14-18 years of age; menopause, pregnancies, breastfeeding, oral contraceptive use, tobacco and alcohol consumption, a BMI of 18.5-24.9, 225-29.9, $\geq 30-34.9$, or (obesity grade II-IV) $\geq 35-40$, lymph node status, metastasis, response to chemotherapy, laboratory tests (hemoglobin (HB), hematocrit (HTO), platelets, leukocytes, urea, serum glutamic oxaloacetic transaminase (SGOT), serum glutamic pyruvic transaminase (SGPT), lactate dehydrogenase (LDH), alkaline phosphatase (ALP), gamma glutamyl transferase (GGT) and glucose). $\mathrm{BC}=$ breast cancer; $\mathrm{SD}=$ standard deviation; $\mathrm{Cl}=$ confidence interval; OR = odds ratio; $\mathrm{DM} 2-\mathrm{AH}=$ diabetes mellitus type 2-arterial hypertension.

Table 4 demonstrates that the null GSTM1 genotype was associated with high plasma levels of glucose $(\mathrm{OR} 0.62,95 \% \mathrm{Cl}=0.42-0.92, \mathrm{P}=0.018)$ and glutamic pyruvic transaminase enzyme (SGPT) (OR $0.50,95 \% \mathrm{Cl}=0.28-0.92, \mathrm{P}=0.027)$, and that the variables listed in Tables 1 and 2 were found to be protective factors. 
Table 3. Genotype distributions of the GSTM1 null polymorphism in patients with BC and healthy controls.

\begin{tabular}{|c|c|c|c|c|c|c|c|}
\hline \multirow{2}{*}{ Genotypes } & \multicolumn{2}{|c|}{ Patients (558) } & \multicolumn{2}{|c|}{ Controls (276) } & \multicolumn{3}{|c|}{ Patients vs Controls } \\
\hline & (N) & $\%$ & (N) & $\%$ & OR & $95 \% \mathrm{Cl}$ & $P$ value \\
\hline No null & (306) & 55 & (172) & 62 & 1 & & \\
\hline Null & (252) & 45 & (104) & 38 & 1.3 & $(1.02-1.8)$ & 0.0 \\
\hline
\end{tabular}

$\mathrm{BC}=$ breast cancer; $\mathrm{OR}=$ odds ratio $\mathrm{Cl}=$ confidence interval.

Table 4. Association of the null genotype of the GSTM1 gene with more than one variable among the general characteristics of the patients with BC.

\begin{tabular}{lllllllll}
\hline & B & SD & Wald & d.f. & P & OR & 95\%Cl \\
\cline { 3 - 8 } & & & & & & & Lower & Upper \\
\hline Glucose & -0.46 & 0.19 & 5.56 & 1 & 0.018 & 0.62 & 0.42 \\
SGPT & -0.67 & 0.30 & 4.87 & 1 & 0.027 & 0.50 & 0.28 \\
Constant & -0.00 & 0.10 & 0.002 & 1 & 0.963 & 0.99 & & 0.92 \\
\hline
\end{tabular}

Variables included in the analysis: dependent: BC patients classified by W/ins-Ins/Ins genotype; independent: personal medical history, menarche ranging from 7-10 years, 11-13 years, or 14-18 years of age; menopause, pregnancies, breastfeeding, oral contraceptive use, tobacco and alcohol consumption, HF, HF type: BC, DM, AH, DM-AH-cancer, a BMI of $18.5-24.9, \geq 25-29.9, \geq 30-34.9$, or $\geq 35->40$, lymph node status, metastasis, response to chemotherapy, laboratory tests (Hemoglobin (HB), hematocrit (HTO), platelets, leukocytes, urea, serum glutamic oxaloacetic transaminase (SGOT), serum glutamic pyruvic transaminase (SGPT), lactate dehydrogenase (LDH), alkaline phosphatase (ALP), gamma glutamyl transferase (GGT) and glucose). $\mathrm{BC}=$ breast cancer; $\mathrm{Cl}=$ confidence interval; $\mathrm{SD}=$ standard deviation; $\mathrm{OR}=$ odds ratio.

Additionally, the null GSTM1 genotype was found to be a protective factor in chemotherapy response in patients with $\mathrm{BC}$ who had high plasma levels of glucose $(\mathrm{OR} 0.56,95 \% \mathrm{Cl}=0.33-0.94$, $P=0.030$, data not shown).

\section{DISCUSSION}

$\mathrm{BC}$ is a multifactorial disease with a complex etiology and is considered a major public health problem in industrialized countries. In Mexico, BC represents one of the leading causes of death in working-age women (Chávarri-Guerra et al. 2012; Miller et al., 2012; Gómez Flores et al., 2013; Ramírez-Patiño et al., 2013; Gallegos-Arreola et al., 2014). These facts are consistent with the observations made in the current study, where the average age of patients with $\mathrm{BC}$ was 54.17 $( \pm 11.6)$ years. A familial history of DM2-AH was found to be risk factor for BC. Wolf et al. (2005) observed that DM2 is a serious health problem that affects more than $7 \%$ of adults in developed countries, that up to $16 \%$ of patients with $\mathrm{BC}$ have $\mathrm{DM} 2$, and that the two major risk factors for DM2, old age and obesity, are also associated with BC.

In our study, when the group was stratified by menopause stage as showing either premenopause or menopause, followed by comparison with the clinical and biochemical characteristics of BC, menarche (14-18 years), lobular carcinoma, luminal B type, and personal history of DM2-AH emerged as risk factors. It has been suggested that the effects of menarche and menopause on BC risk might be due to the function of endogenous ovarian hormones, that would be more relevant for estrogen receptor-positive disease than for estrogen receptor-negative disease and appear to be more relevant for lobular than for ductal tumors (Collaborative Group on Hormonal Factors 
in Breast Cancer, 2012). Wolf et al. (2005) has postulated three mechanisms that could underlie the association of diabetes with BC: activation of the insulin pathway, activation of the insulin-likegrowth-factor pathway, and regulation of endogenous sex hormones. Comparative cohort studies and case-control studies suggest that DM2 might be associated with a $10-20 \%$ excess relative risk of BC. Gestational diabetes mellitus, but not type 1 diabetes, might also be associated with an excess risk of BC. Furthermore, diabetes and its complications can adversely affect cancer therapy and the use of screening, which would affect the outcome of patients with BC.

The genetic regulation of phase II metabolism and its relation to carcinogenesis has been the focus of many investigations. GSTs are a family of important enzymes involved in phase II metabolism and the detoxification of a wide variety of known and suspected carcinogens, including potential mammary carcinogens (Possuelo et al., 2013; Wan et al., 2014). The null GSTM1 polymorphism expresses no GSTM1 enzymatic activity and might present a decreased metabolism of carcinogens, which might accelerate neoplastic processes (Possuelo et al., 2013; Sohail et al., 2013; Wan et al., 2014). Therefore, studies to identify whether the null GSTM1 polymorphism is associated with BC susceptibility have been performed in various populations (Possuelo et al., 2013; Sohail et al., 2013; Wan et al., 2014).

In the present study, we observed similar null GSTM1 genotype frequencies in our control group at those that had been previously reported in Mexico (Pérez-Morales et al., 2011; MartínezRamírez et al., 2013; Sandoval-Carrillo et al., 2014). The genotypic GSTM1 null frequencies were 38 and $45 \%$ in the control group and in patients with BC, respectively, suggesting an association of this allele as a risk factor in BC. Similarly, Wan et al. (2014) detected an association of the null GSTM1 genotype with BC in a meta-analysis study of Chinese populations. Possuelo et al. (2013) also observed a higher frequency of the null GSTM1 polymorphism in patients with BC compared with a control group in a Brazilian population.

In this study, we also observed a protective association of the null GSTM1 genotype in patients with BC with high plasma levels SGPT and glucose. It is known that blood glucose levels are related to SGPT enzyme levels, because glucose is metabolized by the liver enzyme SGPT as an energy and amino acid source for the musculoskeletal system (Xiang et al., 2014). On the other hand, it has been observed that GSTs play an important protective role in preventing the elevation of SGOT and SGPT in the liver, and it is speculated that people with null GSTM1 or GSTT1 genotypes might not detoxify toxic reactive metabolites efficiently and thus might have a higher risk of elevated levels of SGOT and SGPT in the liver (Xiang et al., 2014). Other studies have been inconsistent about the relationship between the null GSTM1 and GSTT1 genotypes and high levels of SGOT and SGPT liver enzymes (Wang et al., 2010; Xiang et al., 2014). A plausible biological explanation for the protective association observed in this study might be that elevated glucose levels mimic the protective association of SGOT observed in patients with BC in this study. Alternatively, some studies have described an association of higher blood glucose levels with the incidence of BC (Wahdan-Alaswad et al., 2013; Xu et al., 2014).

Whereas glucose provides fuel for rapidly dividing cancer cells, insulin is a hormonal stimulator for cellular proliferation (Wahdan-Alaswad et al., 2013). It is also known that hyperglycemia is caused by non-internalization of glucose in the cells of diabetic patients, which contributes to increased ROS generation and oxidative stress which can damage the bases of DNA (LazaldeRamos et al., 2012). Sadi et al. (2013) observed that diabetes causes a significant decrease in the mRNA expression of GSTM1. It is known that the metabolic processes including glucose oxidation, enzymatic glycation of proteins, and subsequent oxidative degradation of glycated proteins can be 
produce ROS in diabetic patients, and these changes subsequently reduce antioxidant defense mechanisms, simultaneously leading to lipid peroxidation and damage to cells. Thus, excessive oxidative stress occurring in the liver due to diabetes mellitus was suggested to result in the down-regulation of GST mu, superoxide dismutase, and catalase gene expression. Accordingly, decreased mRNA expression of the GST mu isoenzyme in patients with diabetes could be due to a decreased half-life of its mRNA because increased oxidative stress might lead to destabilization of mRNA (Sadi et al., 2013).

On other hand, in this study, we observed an association of the null GSTM1 genotype as a protective factor for chemotherapy response in patients with BC who had high plasma levels of glucose. Soto-Quintana et al. (2011) observed a tendency toward better chemotherapy response in patients with the null genotype of GSTT1 and GSTM1 polymorphisms and advanced BC. Additionally, an improved survival in patients with BC carrying the null GSTM1 genotype has been observed, and this has been explained by a better response to chemotherapeutic agents related to more effective cell killing, which in turn is related to the absence of a protective effect of the GSTM1 allele (Lizard-Nacol et al, 1999). Tulsyan et al. (2013) observed a better pharmacogenetic influence of GST polymorphisms on anthracycline-based chemotherapy responses and toxicity in BC. Furthermore, Bai et al. (2012) found a significant association between GSTM1 and GSTP1 gene polymorphisms and clinical outcomes in patients with BC.

Therefore, it is thought that chemotherapy might have a better effect in patients with diabetes carrying the null GSTM1 genotype; both cases might be due to the impossibility of eliminating (phase II) the secondary metabolites of chemotherapy. An increase in ROS production could lead to DNA damage in patients with diabetes who do not exhibit good metabolic control (high glucose); oxidative stress and elevated levels of glucose both reduce the activity of the family of GST enzymes. This effect combined with the null GSTM1 genotype, which cannot affect metabolism of the chemotherapeutic agents, allows the drug to stay in the cell longer, producing a more efficient elimination of tumor cells.

Our results show that the frequencies of the null GSTM1 genotype are significantly different in controls vs patients with BC. A protective association with chemotherapy response was also evident in patients showing high levels of glucose, which might contribute significantly to BC susceptibility in the analyzed sample from a Mexican population. Nevertheless, further studies are required to confirm or reject these conclusions.

\section{ACKNOWLEDGMENTS}

We thank nurses from ${ }^{7}$ Highly Specialized Medical Unit of Gynecology-Obstetrics Hospital, Western National Medical Center, Mexican Institute of Social Security for facilitating sample collection and Western National Medical Center, Mexican Institute of Social Security for support of this project.

\section{REFERENCES}

Bai YL, Zhou B, Jing XY, Zhang B, et al. (2012). Predictive role of GSTs on the prognosis of breast cancer patients with neoadjuvant chemotherapy. Asian Pac. J. Cancer Prev. 13: 5019-5022.

Baranova H, Bothorishvilli R, Canis M, Albuisson E, et al. (1997). Glutathione S-transferase M1 gene polymorphism and susceptibility to endometriosis in a French population. Mol. Hum. Reprod. 3: 775-780.

Chávarri-Guerra Y, Villarreal C, Liedke PE, Knaul F, et al. (2012). Breast cancer in Mexico: a growing challenge to health and the health system. Lancet Oncol. 13: e335-343. 
Collaborative Group on Hormonal Factors in Breast Cancer (2012). Menarche, menopause, and breast cancer risk: individual participant meta-analysis, including 118964 women with breast cancer from 117 epidemiological studies. Lancet Oncol. 13: 1141-1151.

de Aguiar ES, Giacomazzi J, Schmidt AV, Bock H, et al. (2012). GSTM1, GSTT1, and GSTP1 polymorphisms, breast cancer risk factors and mammographic density in women submitted to breast cancer screening. Rev. Bras. Epidemiol. 15: $246-255$.

Duggan C, Ballard-Barbash R, Baumgartner RN, Baumgartner KB, et al. (2013). Associations between null mutations in GSTT1 and GSTM1, the GSTP1 Ile (105) Val polymorphism, and mortality in breast cancer survivors. Springerplus 2; 450.

Gallegos-Arreola MP, Figuera LE, Ramos MC, Salas E, et al. (2014). The association between the 844ins68 polymorphism in the CBS gene and breast cancer. Arch. Med. Sci. 6: 1214-1224.

Gómez Flores-Ramos L, Escoto-De Dios A, Puebla-Pérez AM, Figuera-Villanueva LE, et al. (2013). Association of the tumor necrosis factor-alpha -308G>A polymorphism with breast cancer in Mexican women. Genet. Mol. Res. 12: 5680-5693.

Lazalde-Ramos BP, Zamora-Perez AL, Sosa-Macias M, Guerrero-Velázquez C, et al. (2012). DNA and oxidative damages decrease after ingestion of folic acid in patients with type 2 diabetes. Arch. Med. Res. 43: 476-481.

Lizard-Nacol S, Coudert B, Colosetti P, Riedinger JM, et al. (1999). Glutathione S-transferase M1 null genotype: lack of association with tumour characteristics and survival in advanced breast cancer. Breast Cancer Res. 1: 81-87.

Martínez-Ramírez OC, Pérez-Morales R, Castro C, Flores-Dias A, et al. (2013). Polymorphisms of catechol estrogens metabolism pathway genes and breast cancer risk in Mexican women. Breast 22: 335-343.

McLellan RA, Oscarson M, Alexandrie AK, Seidegård J, et al. (1997). Characterization of a human glutathione S-transferase mu cluster containing a duplicated GSTM1 gene that causes ultrarapid enzyme activity. Mol. Pharmacol. 52: 958-965.

Miller JW, King JB, Joseph DA and Richardson LC (2012). Centers for Disease Control and Prevention (CDC). Breast cancer screening among adult women-behavioral risk factor surveillance system, United States, 2010. MMWR Morb. Mortal. Wkly. Rep. 61: 46-50.

Miller SA, Dykes DD and Polesky HF (1988). A simple salting out procedure for extracting DNA from human nucleated cells. Nucleic Acids Res. 16: 1215.

Pérez-Morales R, Méndez-Ramirez I, Castro-Hernández C, Martínez-Ramirez OC, et al. (2011). Polymorphisms associated with the risk of lung cancer in a healthy Mexican Mestizo population: application of the additive model for cancer. Genet. Mol. Biol. 34: 546-552.

Possuelo LG, Peraça CF, Eisenhardt MF, Dotto ML, et al. (2013). Polymorphisms of GSTM1 and GSTT1 genes in breast cancer susceptibility: a case-control study. Rev. Bras. Ginecol. Obstet. 35: 569-574.

Ramírez-Patiño R, Figuera LE, Puebla-Pérez AM, Delgado-Saucedo JI, et al. (2013). Intron 4 VNTR (4a/b) polymorphism of the endothelial nitric oxide synthase gene is associated with breast cancer in Mexican women. J. Korean Med. Sci. 28 : 1587-1594.

Sadi G, Irtem DI and Güray T (2013). Regulation of glutathione S-transferase Mu with type 1 diabetes and its regulation with antioxidants. Turk. J. Biochem. 38: 106-114.

Sandoval-Carrillo A, Aguila-Duran M, Vázquez-Alaniz F, Castellanos-Juárez FX, et al. (2014). Polymorphisms in the GSTT1 and GSTM1 genes are associated with increased risk of preeclampsia in the Mexican mestizo population. Genet. Mol. Res.13: 2160-2165.

Sanguinetti CJ, Dias Neto E and Simpson AJ (1994). Rapid silver staining and recovery of PCR products separated on polyacrylamide gels. Biotechniques 17: 914-921.

Siegel R, DeSantis C, Virgo K, Stein K, et al. (2012). Cancer treatment and survivorship statistics, 2012. CA Cancer J. Clin. 62: 220-241.

Sohail A, Kanwal N, Ali M, Sadia S, et al. (2013). Effects of glutathione-S-transferase polymorphisms on the risk of breast cancer: a population-based case-control study in Pakistan. Environ. Toxicol. Pharmacol. 35: 143-153.

Soto-Quintana O, Cabrera-Galeana P, Téllez-Trevilla G, Barrera-Franco JL, et al. (2011). Relationship of polymorphisms of glutathione S-transferase GSTT1 and GSTM1 with the response to chemotherapy in Mexican women with advanced breast cancer. JCT 2: 354-361.

Tulsyan S, Chaturvedi P, Agarwal G, Lal P, et al. (2013). Pharmacogenetic influence of GST polymorphisms on anthracyclinebased chemotherapy responses and toxicity in breast cancer patients: a multi-analytical approach. Mol. Diagn. Ther. 17: 371-379.

Wahdan-Alaswad R, Fan Z, Edgerton SM, Liu B, et al. (2013). Glucose promotes breast cancer aggression and reduces metformin efficacy. Cell Cycle 12: 3759-3769.

Wan G, Li F, Li W, Sun J, et al. (2014). Glutathione S-transferase M1 polymorphism and susceptibility to breast cancer in Chinese population: a meta-analysis. Zhonghua Bing Li Xue Za Zhi. 43: 158-162.

Wang T, Yu HT, Wang W, Pan YY, et al. (2010). Genetic polymorphisms of cytochrome P450 and glutathione S-transferase associated with antituberculosis drug-induced hepatotoxicity in Chinese tuberculosis patients. J. Int. Med. Res. 38: $977-986$. 
WHO Expert Consultation (2004). Appropriate body-mass index for Asian populations and its implications for policy and intervention strategies. Lancet 363: 157-163.

Wolf I, Sadetzki S, Catane R, Karasik A, et al. (2005). Diabetes mellitus and breast cancer. Lancet Oncol. 6: 103-111.

Xiang Y, Ma L, Wu W, Liu W, et al. (2014). The incidence of liver injury in Uyghur patients treated for TB in Xinjiang Uyghur autonomous region, China, and its association with hepatic enzyme polymorphisms NAT2, CYP2E1, GSTM1 and GSTT1. PLoS One 9: e85905.

Xu CX, Zhu HH and Zhu YM (2014). Diabetes and cancer: Associations, mechanisms, and implications for medical practice. World J. Diabetes 5: 372-380. 